ERRATA

\title{
Erratum: Gene-editing nucleases
}

Monya Baker

Nat. Methods 9, 23-26 (2012); published online 28 December 2011; corrected after print 30 January 2012.

In the version of this article initially published, a scientist's last name and affiliation were incorrect. The error has been corrected in the HTML and PDF versions of the article. 